九州大学学術情報リポジトリ

Kyushu University Institutional Repository

\title{
Discovering Frequent Substructures in Large Unordered Trees
}

Asai, Tatsuya

Kyushu University

Arimura, Hiroki

Kyushu University

Uno, Takeaki

National Institute of Informatics

Nakano, Shin-ichi

Gunma University

http://hdl. handle. net/2324/3055

出版情報 : DOI Technical Report. 216，2003-06. Department of Informatics, Kyushu University バージョン:

権利関係: 
DOI-TR-CS 216, Department of Informatics, Kyushu University, June, 2003

ftp://ftp.i.kyushu-u.ac.jp/pub/tr/trcs216.pdf (Submitting)

\title{
Discovering Frequent Substructures in Large Unordered Trees
}

\author{
Tatsuya Asai ${ }^{1}$, Hiroki Arimura ${ }^{1}$, Takeaki Uno ${ }^{2}$, and Shin-ichi Nakano ${ }^{3}$ \\ 1 Kyushu University, Fukuoka 812-8581, JAPAN \\ \{t-asai, arim\}@i.kyushu-u.ac.jp \\ 2 National Institute of Informatics, Tokyo 101-8430, JAPAN \\ uno@nii.jp \\ 3 Gunma University, Kiryu-shi, Gunma 376-8515, JAPAN \\ nakano@cs.gunma-u.ac.jp
}

In this paper, we study a data mining problem of discovering frequent substructures in a large collection of semi-structured data, where both of the patterns and the data are modeled by labeled unordered trees. An unordered tree is a directed acyclic graph with a specified node called the root, and all nodes but the root have at most one parent. Each node is labeled by a symbol drawn from an alphabet. Such unordered trees can be seen as either a generalization of itemsets in relational databases or an efficient specialization of attributed graphs in graph mining. They are also useful in various applications such as analysis of chemical compounds and mining hyperlink structures in Web. Introducing novel definitions of the support and the canonical form for unordered trees, we present an efficient algorithm called Unot that computes all labeled unordered trees appearing in a collection of data trees with frequency above a user-specified threshold. We prove that the algorithm enumerates each frequent pattern $T$ in $O\left(k b^{2} n\right)$ per pattern, where $k$ is the size of $T, b$ is the branching factor of the data tree, and $n$ is the total number of occurrences of $T$ in the data trees. The keys of the algorithm are efficient enumerating all unordered trees in canonical form and incrementally computation of the occurrences based on a powerful design technique known as the reverse search.

Correspondence:

Tatsuya Asai

Department of Informatics, Kyushu University, 6-10-1 Hakozaki Higashi-ku, Fukuoka 812-8581, JAPAN

E-mail: t-asai@i.kyushu-u.ac.jp

phone: +81-92-642-2697, fax: +81-92-642-2698 


\section{Introduction}

By rapid progress of network and storage technologies, huge amounts of electronic data has been available in various enterprises and organizations. These weakly-structured data are well modeled by graph or trees, where a data object is represented by a nodes and a connection or relationships between objects are encoded by an edge between them. There have been increasing demands for efficient methods for discovering patterns in large collections of graph and tree structures[1, 4, 5, 7-10,15-18].

In this paper, we present an efficient algorithm for discovering frequent substructures in a large graph structured data, where both of the patterns and the data are modeled by labeled unordered trees. A labeled unordered tree is a rooted directed acyclic graph, where all but the root node have exactly one parent and each node is labeled by a symbol drawn from an alphabet (Fig. 1). Such unordered trees can be seen as either a generalization of labeled ordered trees extensively studied in semi-structured data mining $[1,4,5,10,13,16,18]$, or as an efficient specialization of attributed graphs in graph mining researches[7-9, $15,17]$. They are also useful in modeling various types of unstructured or semistructured data such as chemical compounds, dependency structure in discourse analysis and the hyperlink structure of Web sites.

On the other hand, difficulties arise in discovery of trees and graphs such as the combinatorial explosion of the number of possible patterns, the isomorphism problem for many semantically equivalent patterns. Also, there are other difficulties such as the computational complexity of detecting the embeddings or occurrences in trees. We tackle these problems by introduce a novel definitions of the support and the canonical form for unordered trees, and by developing techniques for efficiently enumerating all unordered trees in canonical form without duplicates and for incrementally computing the embeddings of each patterns in data trees. Interestingly, these techniques can be seen as instances of the reverse search technique, known as a powerful design tool for combinatorial enumeration problems [6,14].

Combining these techniques, we present an efficient algorithm Unot (Unot is Not for Ordered Trees) that computes all labeled unordered trees appearing in a collection of data trees with frequency above a user-specified threshold. The algorithm Unot has a provable performance in terms of the output size unlike other graph mining algorithm presented so far. It enumerates each frequent pattern $T$ in $O\left(k b^{2} n\right)$ per pattern, where $k$ is the size of $T, b$ is the branching factor of the data tree, and $n$ is the total number of occurrences of $T$ in the data trees. Although we present only theoretical results in this manuscript, we are implementing a prototype of the algorithm and will run computer experiments on synthesized and real-world data to give empirical evaluation of the algorithm in the revised version.

This paper is organized as follows. In Section 2, we prepare basic definitions and results on unordered trees and introduce our data mining problem. In Section 3, we define the canonical form for the unordered trees. In Section 4, we show an efficient algorithm for enumerating unordered trees in canonical form in $O(1)$ time per tree. In Section 5, we develop an incremental algorithm for updating an embedding occurrence list. Combining these techniques, we present an efficient algorithm Unot for discovering frequent unordered trees in a given database of unordered trees. In Section 6, we conclude the results. 


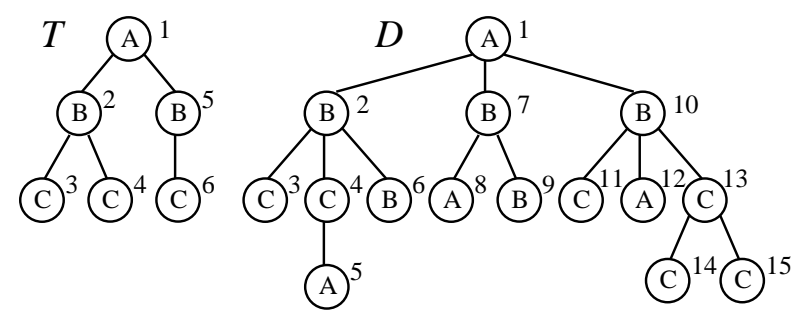

Fig. 1. A data tree $D$ and a pattern tree $T$

\section{Preliminaries}

In this section, we give basic definitions on unordered trees according to [2] and then introduce our data mining problems. For a set $A,|A|$ denotes the size of $A$. For a binary relation $R \subseteq A^{2}$ on $A, R^{*}$ denotes the reflexive transitive closure of $R$.

\subsection{The Model of Semi-structured data}

We introduce the class of labeled unordered trees as a model of semi-structured data and patterns according to $[2-4,12]$. Let $\mathcal{L}=\left\{\ell, \ell_{1}, \ell_{2}, \ldots\right\}$ be a countable set of labels with a total order $\leq_{\mathcal{L}}$ on $\mathcal{L}$.

A labeled unordered tree (an unordered tree, for short) is a directed acyclic graph $T=(V, E, r$, label $)$, with a distinguished node $r$ called the root, satisfying the followings. $V$ is a set of nodes, called the domain, and $E \subseteq V \times V$ is a set of edges. The mapping label $: V \rightarrow \mathcal{L}$ is called a labeling function. For every node $v \in V$, there is a unique path $U P(v)=\left(v_{0}=r, v_{1}, \ldots, v_{d}\right)(d \geq 0)$ from the root $r$ to $v$. Then, the depth of $v$ is $\operatorname{dep}(v)=d$.

Let $u$ and $v$ be nodes. If $(u, v) \in E$ then we say that $u$ is a parent of $v$, or $v$ is a child of $u$. If there is a path from $u$ to $v$, then we say that $u$ is an ancestor of $v$, or $v$ is a descendant of $u$. A leaf is a node having no child. For a node $v$, we denote by $T(v)$ the subtree of $T$ rooted at $v$, the subgraph of $T$ induced in the set of all descendants of $v$. The size of $T$ is defined as the number of its nodes $|T|=|V|$. We define the empty tree as a special tree $\perp$ of size 0 .

Example 1. In Fig. 1, we show examples of labeled unordered trees $T$ and $D$ on alphabet $\mathcal{L}=\{A, B, C\}$ with the total ordering $A>B>C$. In the tree $T$, the root is 1 labeled with $A$ and the leaves are 3,4 , and 6 . The subtree $T(2)$ at node 2 consists of nodes $2,3,4$. The size of $T$ is $|T|=6$.

We use a labeled ordered tree as a representation of a labeled unordered tree. A labeled ordered tree (an ordered tree, for short) is an unordered tree with the left-to-right ordering among the children of each nodes. Formally, a labeled ordered tree is a 5 -tuple $T=(V, E, B, r$, label $)$, where $V, E$, label, and $r$ are the node sets, the edge sets, the labeling function of $V$, and the root of $T$. The binary relation $B \subseteq V \times V$ is the left-to-right ordering among the children. If $\left(v_{1}, v_{2}\right) \in B$ then we say that $v_{1}$ precedes $v_{2}$, or $v_{2}$ follows $v_{1}$. Let $R M B(T)=\left(r_{0}, \ldots, r_{c}\right)(c \geq 0)$ be the path from the root $r$ to the rightmost leaf of $T$. The path $R M B(T)$ is called the rightmost branch of $T$. We denote 
by $\operatorname{rml}(T)=k$ the rightmost leaf of $T$. See [4] for more definitions on labeled ordered trees.

We denote by $\mathcal{U}$ and $\mathcal{T}$ the class of unordered trees and the class of ordered trees over $\mathcal{L}$, respectively. For a labeled unordered tree $T=(V, E, r$, label $)$, we write $V_{T}, V_{E}, r_{T}$ and $l_{a b e l}$ for $V, E, r$ and label if it is clear from the context.

\subsection{Tree Matching}

In this paper, we give the semantics of a labeled unordered trees by the tree matching, which is popular in computational learning theory, semi-structured data mining, and pattern matching $[3,4]$.

Let $T=\left(V_{T}, E_{T}, r_{T}\right.$, label $\left._{T}\right) \in \mathcal{U}$ and $D=\left(V_{D}, E_{D}, r_{D}\right.$, label $\left._{D}\right) \in \mathcal{U}$ be unordered trees. We call $T$ and $D$ the pattern tree and the data tree, respectively. Then, we say that the pattern tree $T$ occurs in the data tree $D$ as an unordered tree if there is a mapping $\varphi: V_{T} \rightarrow V_{D}$ satisfying the following (1)-(3) for every $x, y \in V_{T}$ :

(1) $\varphi$ is one-to-one, i.e., $x \neq y$ implies $\varphi(x) \neq \varphi(y)$.

(2) $\varphi$ preserves the parent relation, i.e., $(x, y) \in E_{T}$ iff $(\varphi(x), \varphi(y)) \in E_{D}$.

(3) $\varphi$ preserves the labels, i.e., $\operatorname{label}_{T}(x)=\operatorname{label}_{D}(\varphi(x))$.

Then, the mapping $\varphi$ is called a matching from $T$ into $D$.

\subsection{Patterns and their Occurrences}

For a nonnegative integer $k$, a $k$-unordered pattern ( $k$-pattern, for short) is a labeled unordered tree having exactly $k$ nodes, that is, $V_{T}=\{1, \ldots, k\}$. Assume that the root $r$ of a $k$-pattern is always node 1 .

A database on $\mathcal{L}$ is a finite collection $\mathcal{D}=\left\{D_{1}, \ldots, D_{n}\right\} \subseteq \mathcal{U}$, where each $D_{i} \in \mathcal{D}$ is an unordered tree on $\mathcal{L}$ called a data tree, and $V_{D_{i}} \cap V_{D_{j}}=\emptyset$ for every $1 \leq i, j \leq n(i \neq j)$. Then, we denote by $V_{\mathcal{D}}$ the set of all nodes in the data trees in $\mathcal{D}$, and then we extend the notion of the matching by a pattern $T \in \mathcal{U}$ occurs in $\mathcal{D}$ with a mapping $\varphi: V_{T} \rightarrow V_{\mathcal{D}}$ if $\varphi$ is a matching from $T$ to some $D \in \mathcal{D}$. Let $\mathcal{M}^{\mathcal{D}}(T)$ the set of all matchings from $T$ into $\mathcal{D}$.

Definition 1. Let $k \geq 1$ be any positive integer and $T=(V, E, r$, label $)$ be a $k$-unordered pattern. Assume that we have a matching $\varphi: V_{T} \rightarrow V_{D} \in \mathcal{M}^{\mathcal{D}}(T)$ from $T$ into $\mathcal{D}=\left\{D_{i}\right\}_{i}$. Then, we define four types of occurrences of $U$ as follows:

1. The total occurrence of $T$ is the $k$-tuple $\operatorname{Toc}(\varphi)=\langle\varphi(1), \ldots, \varphi(k)\rangle \in\left(V_{D}\right)^{k}$.

2. The embedding occurrence of $T$ is the set $\operatorname{Eoc}(\varphi)=\{\varphi(1), \ldots, \varphi(k)\} \subseteq V_{D}$.

3. The root occurrence of $T: \operatorname{Roc}(\varphi)=\varphi(1) \in V_{D}$

4. The document occurrence of $T$ is the index $\operatorname{Doc}(\varphi)=i \operatorname{such}$ that $\operatorname{Eoc}(\varphi) \subseteq$ $V_{D_{i}}$ for some $1 \leq i \leq|\mathcal{D}|$.

Example 2. In Fig. 1, we see that the pattern tree $S$ has eight total occurrences $\varphi_{1}=\langle 1,2,3,4,10,11\rangle, \varphi_{2}=\langle 1,2,4,3,10,11\rangle, \varphi_{3}=\langle 1,2,3,4,10,13\rangle$, $\varphi_{4}=\langle 1,2,4,3,10,13\rangle, \varphi_{5}=\langle 1,10,11,13,2,3\rangle, \varphi_{6}=\langle 1,10,13,11,2,3\rangle, \varphi_{7}=$ $\langle 1,10,11,13,2,4\rangle$, and $\varphi_{8}=\langle 1,10,13,11,2,4\rangle$ in the data tree $D$, where we identify the matching $\varphi_{i}$ and $\operatorname{Toc}\left(\varphi_{i}\right)$. On the other hand, there are four embedding occurrences $\operatorname{Eoc}\left(\varphi_{1}\right)=\operatorname{Eoc}\left(\varphi_{2}\right)=\{1,2,3,4,10,11\}, \operatorname{Eoc}\left(\varphi_{3}\right)=\operatorname{Eoc}\left(\varphi_{4}\right)=$ $\{1,2,3,4,10,13\}, \operatorname{Eoc}\left(\varphi_{5}\right)=\operatorname{Eoc}\left(\varphi_{6}\right)=\{1,2,3,10,11,13\}$, and $\operatorname{Eoc}\left(\varphi_{7}\right)=$ $\operatorname{Eoc}\left(\varphi_{8}\right)=\{1,2,4,10,11,13\}$, and there is one root occurrence $\varphi_{1}(1)=\varphi_{2}(1)=$ $\cdots=\varphi_{8}(1)=1$ of $T$ in $D$. 
Now, we analyze the relationship among the above definitions of the occurrences by introducing an ordering $\geq_{\text {occ }}$ on the definitions. For a type of occurrences $\tau, \pi \in\{T o c, E o c, R o c, D o c\}$, we say $\pi$ is stronger than or equal to $\tau$, denoted by $\pi \geq_{\text {occ }} \tau$, iff for every matchings $\varphi_{1}, \varphi_{2} \in \mathcal{M}^{\mathcal{D}}(T)$ from $T$ to $\mathcal{D}, \pi\left(\varphi_{1}\right)=\pi\left(\varphi_{2}\right)$ implies $\tau\left(\varphi_{1}\right)=\tau\left(\varphi_{2}\right)$. Then, we have the following linear ordering on classes of occurrences.

Lemma 1. $T o c \geq_{\text {occ }} E o c \geq_{\text {occ }} R o c \geq_{\text {occ }}$ Doc.

For an unordered pattern $T \in \mathcal{U}$, we denote by $T O^{\mathcal{D}}(T), E O^{\mathcal{D}}(T), R O^{\mathcal{D}}(T)$, and $D O^{\mathcal{D}}(T)$ the set of the total occurrences, the embedding occurrences, the root occurrences, and the document occurrences of $T$ in $\mathcal{D}$, respectively. Furthermore, we show the upper and the lower bounds of the relative size of the occurrences among $T O^{\mathcal{D}}(T), E O^{\mathcal{D}}(T)$, and $R O^{\mathcal{D}}(T)$ as follows.

Lemma 2. Let $\mathcal{D}$ be a database and $T$ be a pattern. Then,

$$
\frac{\left|T O^{\mathcal{D}}(T)\right|}{\left|E O^{\mathcal{D}}(T)\right|}=k^{\Theta(k)} \quad \text { and } \quad \frac{\left|E O^{\mathcal{D}}(T)\right|}{\left|R O^{\mathcal{D}}(T)\right|}=\Theta\left(n^{k}\right)
$$

over all pattern $T \in \mathcal{U}$ and all databases $\mathcal{D} \in 2^{\mathcal{U}}$ satisfying $k \leq$ cn for some $0<c<1$, where $k$ is the size of $T$ and $n$ is the size of a database.

Proof. The upperbounds are easy. Therefore, we show the lowerbound of $\gamma=$ $\left|T O^{\mathcal{D}}(T)\right| /\left|E O^{\mathcal{D}}(T)\right|$ as follows. Consider a labeled unordered tree $T$ with the domain $V_{T}=\{0,1, \ldots, k\}$ such that the root is 0 and the leaves are $\{1, \ldots, k\}$. All nodes have the same label $a$. For the database $\mathcal{D}=\{T\}$, $T$ has $k$ ! $=$ $k^{\Theta(k)}$ distinct total occurrences $\left\langle 0, v_{1}, \ldots, v_{k}\right\rangle$, where $\left\langle v_{1}, \ldots, v_{k}\right\rangle$ is any permutation of $\langle 1, \ldots, k\rangle$. On the other hand, there exists exactly one embedding occurrence $\{0,1, \ldots, k\}$, and this shows $\gamma=k^{\Omega(k)}$. The lowerbound of $\delta=\left|E O^{\mathcal{D}}(T)\right| /\left|R O^{\mathcal{D}}(T)\right|$ can be shown by the same pattern $T$ and the data tree with the root 0 and the leaves $\{1, \ldots, n\}(n>k>0)$. Then, $T$ has exactly ${ }_{n} C_{k}$ embeddings in $\mathcal{D}$, where ${ }_{n} C_{k} \geq(n-k)^{k} \geq(1-c)^{k} n^{k}=n^{\Omega(k)}$.

By the lemma, the embedding occurrences $E O^{\mathcal{D}}(T)$ is exponentially smaller than $T O^{\mathcal{D}}(T)$. For various types of tree mining algorithms, the running time and the space that the algorithm used heavily depend on the maximum and the expected sizes of the occurrences of a pattern $T$. Thus, we have to carefully select the type of occurrences considering the tradeoff between the expressiveness and the efficiency.

Obviously, the total occurrence $\operatorname{Toc}(\varphi)$ contains the same information to the matching function $\varphi$ itself, while it is too costly to maintain. In the application point of view, the document occurrence $\operatorname{Doc}(\varphi)$ is most desirable, but contains too few information to incrementally compute. In the previous work on ordered tree mining [4], we adopted the root occurrence $\operatorname{Roc}(\varphi)$ with the rightmost leaf occurrences as the standard class of occurrences. In this paper, we use the embedding occurrences $\operatorname{Eoc}(\varphi)$ for unordered trees.

Let $\tau \in\{T o c, E o c, R o c, D o c\}$ be types of the occurrences, $U$ be a pattern and $\mathcal{D}$ be a database. Then, the $\tau$-count of $U$ in $\mathcal{D}$ is $\left|\tau^{\mathcal{D}}(U)\right|$ and the (relative) $\tau$ frequency is the ratio $\left|\tau^{\mathcal{D}}(T)\right| /|\mathcal{D}|$. Let $0<\sigma<1$ be any positive number, called the minimum frequency threshold. A pattern $T$ is $\tau$-frequent with the minimum frequency $\sigma$ (or frequent) if $T$ has the relative $\tau$-frequency no smaller than $\sigma$. Then, we state our data mining problems we will consider in this paper as follows. Recall that $\tau \in\{T o c, E o c, R o c, D o c\}$ is a type of the occurrences. 

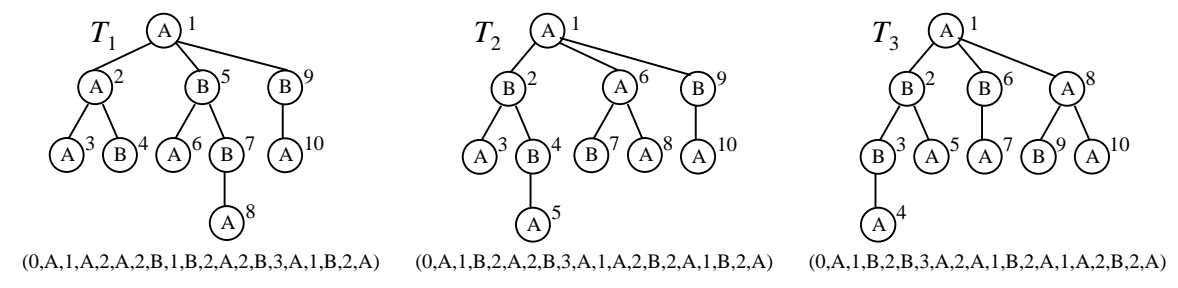

Fig. 2. The depth-label sequences of labeled ordered trees

Frequent Unordered Tree Discovery with Occurrence Type $\tau$ Given a database $\mathcal{D} \subseteq \mathcal{U}$ and a minimum frequency threshold $0 \leq \sigma \leq 1$, find all the $\tau$-frequent patterns satisfying $\left|\tau^{\mathcal{D}}(U)\right| \geq|\mathcal{D}| \sigma$.

In this paper, we study only the frequent unordered tree discovery problem with embedding occurrences. However, it is not so difficult to modify the results of this paper for other types of problems.

In some applications such as the analysis of chemical compounds, it is useful to enumerate all the embedded substructures in a given data graph. The following problem deals with a special case where the data graph restricted to an unordered tree.

\section{Substructure Discovery Problem for Unordered Trees}

Given a labeled unordered tree $D \in \mathcal{U}$, find all the labeled unordered trees $T \in \mathcal{U}$ embedded in $D$, that is, $T$ occurs in $D$ at least once.

Finally, we discuss the representation of a data tree in implementation. Throughout this paper, we adopt the first-child next-sibling representation [2] for ordered trees as well as unordered trees. In this representation, each node $v$ has two pointers firstchild $(v)$ and nextsibling $(v)$ to its first child and the next sibling, respectively. If there are no such nodes, the pointers are set to be null.

\section{Canonical Representation for Unordered Trees}

In this section, we give the canonical representation for unordered tree patterns according to Nakano and Uno [12].

\subsection{Depth Sequence of a Labeled Unordered Tree}

We use labeled ordered trees as the representation of labeled unordered trees. First, we introduce some technical definitions on ordered trees. Throughout this paper, we assume that any labeled ordered tree $T=(V, E, B, r$, label $)$ of size $k$ satisfies that the node set is $V=\{1, \ldots, k\}$ and the elements in $V$ are numbered consecutively in preorder. Obviously, the root of $T$ is $r=1$ and the rightmost leaf is $r m l(T)=k$. We denote by $\psi(T)=(V, E, r$, label $)$ the unordered tree obtained from $T$ by ignoring the left-to-right order $B$ over the children. Two ordered trees $T_{1}$ and $T_{2} \in \mathcal{T}$ are equivalent each other as unordered trees, denoted by $T_{1} \equiv T_{2}$, if $\psi\left(T_{1}\right)=\psi\left(T_{2}\right)$ holds.

Let $T=(V, E, B, r$, label $)$ be a labeled ordered tree of size $k$. A depth-label pair is $(d, \ell)$, where $d \in \mathbf{N}$ and $\ell \in \mathcal{L}$. For the list $(1,2, \ldots, k)$ of the nodes of $T$ 
in preorder, let $\operatorname{dep}(i)$ be the depth of the $i$-th node $i$ for $i=1, \ldots, k$. Then, the sequence

$$
C(T)=\left((\operatorname{dep}(1), \operatorname{label}(1)), \ldots,(\operatorname{dep}(k), \operatorname{label}(k)) \in(\mathbf{N} \times \mathcal{L})^{*}\right.
$$

is called the depth-label sequence of $T[5,11,18]$.

Example 3. In Fig. 2, we present examples of the depth-label sequences. These trees $T_{1}, T_{2}$, and $T_{3}$ are equivalent as unordered trees, but not as ordered trees.

Next, we introduce the total ordering $\geq$ over depth-label sequences as follows. For depth-label pairs $\left(d_{1}, \ell_{1}\right)$ and $\left(d_{2}, \ell_{2}\right),\left(d_{1}, \ell_{1}\right)>\left(d_{2}, \ell_{2}\right)$ iff either (i) $d_{1}>d_{2}$ or (ii) $d_{1}=d_{2}$ and $\ell_{1}>\ell_{2}$. We extend this ordering to the lexicographic ordering over depth-label sequences. Let $T_{1}$ and $T_{2}$ be two labeled ordered trees, and $C\left(T_{1}\right)=\left(x_{1}, \ldots, x_{m}\right)$ and $C\left(T_{2}\right)=\left(y_{1}, \ldots, y_{m}\right)$ be their depth-label sequences $(m, n \geq 0)$. We say $C\left(T_{1}\right)$ is heavier than $C\left(T_{2}\right)$, denoted by $C\left(T_{1}\right) \geq_{\operatorname{lex}} C\left(T_{2}\right)$, if there exists some $k$ such that (i) $x_{i}=y_{i}$ for each $i=1, \ldots, k-1$ and (ii) either $x_{k}>y_{k}$ or $m>k-1=n$.

Now, we give the canonical representation for labeled unordered trees as follows.

Definition 2 (Nakano and Uno [12]). Let $\mathcal{L}$ be an alphabet and $T$ be a labeled unordered tree over $\mathcal{L}$. Then, the canonical representation of $T$, denoted by $C R(T)$, is the labeled ordered tree $S \in \mathcal{T}$ over $\mathcal{L}$ such that its depth-label sequence $C(S)$ is the heaviest sequence in the equivalence class $\left\{\hat{C}\left(S^{\prime}\right) \mid S^{\prime} \in\right.$ $\left.\mathcal{T}, S^{\prime} \equiv S\right\}$.

The next lemma gives a characterization of the canonical representations for unordered trees [12].

Lemma 3 (Left-heavy condition [12]). A labeled ordered tree $T$ is the canonical representation of some unordered tree iff $T$ is left-heavy, that is, for any node $v_{1}, v_{2} \in V,\left(v_{1}, v_{2}\right) \in B$ implies $C\left(T\left(v_{1}\right)\right) \geq_{\operatorname{lex}} C\left(T\left(v_{2}\right)\right)$.

Example 4. Three ordered trees $T_{1}, T_{2}$, and $T_{3}$ in Fig. 2 represents the same unordered tree. Among them, $T_{1}$ is left-heavy and thus it is the canonical representation of a labeled unordered tree under the assumption that $A>B>C$. On the other hand, $T_{2}$ is not canonical since the depth-label sequence $C\left(T_{2}(2)\right)=$ $(1 B, 2 A, 2 B, 3 A)$ is lexicographically smaller than $C\left(T_{2}(6)\right)=(1 A, 2 B, 2 A)$ and this violates the left-heavy condition. $T_{3}$ is not canonical since $B<A$ implies $C\left(T_{3}(3)\right)=(2 B, 3 A)<_{\text {lex }} C\left(T_{3}(5)\right)=(2 A)$.

By the above construction, we have assigned the unique labeled ordered tree in $\mathcal{T}$ to each labeled unordered tree in $\mathcal{U}$, and they can be used as the canonical representations for trees in $\mathcal{U}$. A labeled ordered tree $S$ is in the canonical form or a canonical representation if $S=C R(T)$ for some labeled unordered tree $T \in \mathcal{U}$. We denote by $\mathcal{C}$ the class of the canonical representations of labeled unordered trees over $\mathcal{L}$. In what follows, we identify a labeled ordered tree $S \in \mathcal{T}$ and its depth-label sequence if it is clear from context. Thus, we may write $S>_{\text {lex }} T$ for ordered trees $S, T \in \mathcal{T}$. 


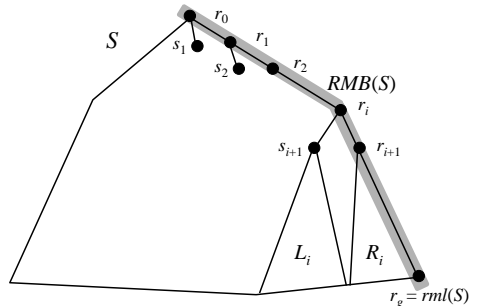

Fig. 3. Notions on a canonical representation
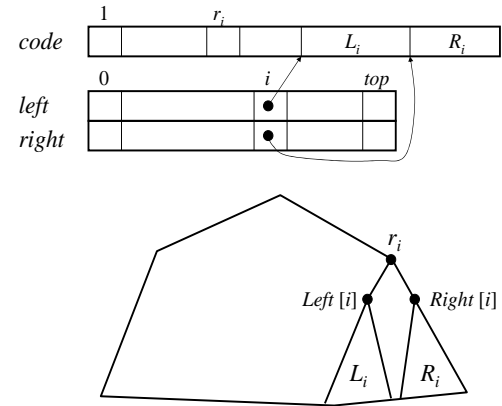

Fig. 4. Data structure for a pattern

\subsection{The Reverse Search Principle and the Rightmost Expansions}

The reverse search is a general scheme for designing efficient algorithm for hard enumeration problems $[6,14]$. In reverse search, we define the parent-child relation $P \subseteq \mathcal{S} \times \mathcal{S}$ on the solution space $\mathcal{S}$ of the problem so that each solution $X$ has the unique parent $P(X)$. Since this relation forms a search tree over $\mathcal{S}$, we enumerate the solutions starting from the root solutions and by computing the children for the solutions. Iterating this process, we can generate all the solutions without duplicates.

Let $T$ be a labeled ordered tree having at least two nodes. We denote by $P(T)$ the unique labeled ordered tree derived from $T$ by removing the rightmost leaf $\operatorname{rml}(T)$. We say $P(T)$ is the parent tree of $T$ or $T$ is a child tree of $P(T)$. The following lemma is crucial to our result.

Lemma 4 (Nakano and Uno [12]). For any labeled ordered tree $T \in \mathcal{T}$, if $T$ is in canonical form then so is its parent $P(T)$, that is, $T \in \mathcal{C}$ implies $P(T) \in \mathcal{C}$.

Proof. For a left-heavy tree $T \in \mathcal{C}$, the operation to remove the rightmost leaf from $T$ does not violate the left-heavy condition of $T$. It follows from Lemma 3 that the lemma holds.

Definition 3 (Rightmost expansion $[4,11,18]$ ). Let $S \in \mathcal{T}$ be a labeled ordered tree on $\mathcal{L}$. Then a labeled ordered tree $T \in \mathcal{T}$ is the rightmost expansion of $S$ if $T$ is obtained from $S$ by attaching the new node $v$ as the rightmost child of a node on the rightmost branch $R M B(S)$ of $S$. If $(\operatorname{dep}(v), \operatorname{label}(v))=(d, \ell)$ then we call $T$ the $(d, \ell)$-expansion of $S$. We define the $(0, \ell)$-expansion of $\perp$ to be the single node tree with label $\ell$.

Since newly attached node $v$ is the last node in preorder on $T$, we denote the $(d, \ell)$-expansion of $S$ by $S \cdot(d, \ell)$. We sometimes write $\boldsymbol{v}=(\operatorname{dep}(v), \operatorname{label}(v))$.

If we can compute the set of the child trees of a given labeled ordered tree $S \in \mathcal{T}$ then we can enumerate all the labeled ordered trees in $\mathcal{T}$. The method is called the rightmost expansion and has been independently studied in $[4,11,18]$.

\section{Mining Frequent Unordered Tree Patterns}

In this section, we present an efficient algorithm Unot for solving the frequent unordered tree pattern discovery problem w.r.t. the embedding occurrences. 


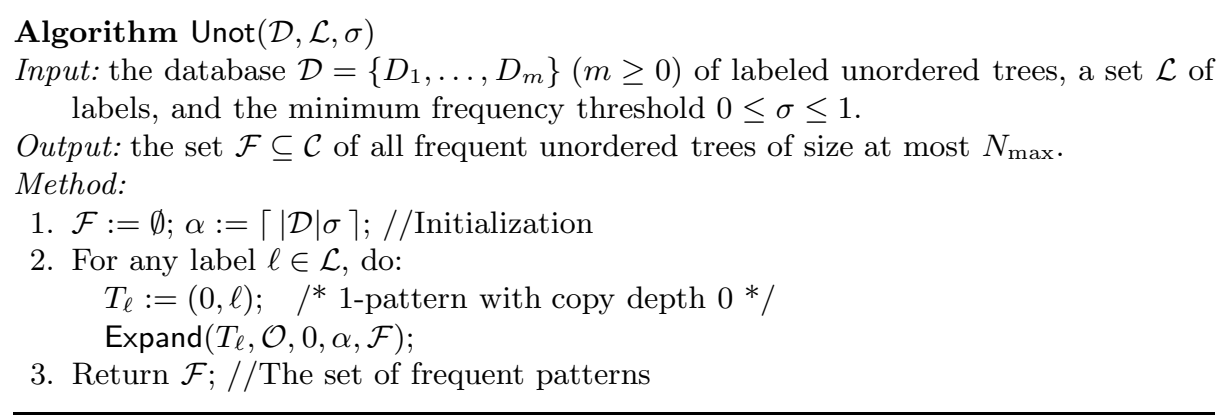

Fig. 5. An algorithm for discovering all frequent unordered trees

\subsection{Overview of the Algorithm}

In Fig. 5, we show our algorithm Unot for finding all the canonical representations for frequent unordered trees in a given database $\mathcal{D}$. A key of the algorithm Unot is efficient enumeration of all the canonical representations, which is implemented by the subprocedures FindAllChildren in Fig. 5 to run in $O(1)$ time per pattern. Another key is incremental computation of their occurrences. This is implemented by the subprocedure UpdateOcc in Fig. 8 to run in $O\left(b k^{2} n\right)$ time per pattern, where $b$ is the maximum branching factor in $\mathcal{D}, k$ is the maximum pattern size, $m$ is the umber of embedding occurrences. We give the detailed descriptions of these procedures in the following subsections.

\subsection{Enumerating Unordered Trees}

First, we prepare some notations (Fig. 3). Let $T$ be a labeled ordered tree with the rightmost branch $R M B(T)=\left(r_{0}, r_{1}, \ldots, r_{g}\right)$. For every $i=0,1, \ldots, g$, if $r_{i}$ has two or more children then we denote by $s_{i+1}$ the child of $r_{i}$ preceding $r_{i+1}$, that is, $s_{i+1}$ is the second rightmost child of $r_{i}$. Then, we call $L_{i}=T\left(s_{i+1}\right)$ and $R_{i}=T\left(r_{i+1}\right)$ the left and the right tree of $r_{i}$. If $r_{i}$ has exactly one child $r_{i+1}$ then we define $L_{i}=\top_{\infty}$, where $\top_{\infty}$ is a special tree such that $\top_{\infty}>_{\text {lex }} S$ for any $S \in \mathcal{T}$. For a pattern tree $T \in \mathcal{T}$, we sometimes write $L_{i}^{(T)}$ and $R_{i}^{(T)}$ for $L_{i}$ and $R_{i}$ by indicating the pattern tree $T$.

By Lemma 3, an ordered tree is in canonical form iff it is left-heavy. The next lemma claims that the algorithm only checks the left trees and right trees of nodes on the rightmost branch to check if the tree is in canonical form.

Lemma 5 (Nakano and Uno [12]). Let $S \in \mathcal{C}$ be a canonical representation and $T$ be a child tree of $S$ with the rightmost branch $\left(r_{0}, \ldots, r_{g}\right)$, where $g \geq 0$. Then, $T$ is in canonical form iff $L_{i} \geq_{\operatorname{lex}} R_{i}$ holds in $T$ for every $i=0, \ldots, g-1$.

Let $T$ be a labeled ordered tree with the rightmost branch $R M B(T)=$ $\left(r_{0}, r_{1}, \ldots, r_{g}\right)$. We say $C\left(L_{i}\right)$ and $C\left(R_{i}\right)$ have a disagreement at the position $j$ if $j \leq \min \left(\left|C\left(L_{i}\right)\right|,\left|C\left(R_{i}\right)\right|\right)$ and the $j$-th components of $C\left(L_{i}\right)$ and $C\left(R_{i}\right)$ are different pairs.

Suppose that $T$ is in canonical form. During a sequence of rightmost expansions to $T$, the $i$-th right tree $R_{i}$ grows as follows.

1. Firstly, when a new node $v$ is attached to $r_{i}$ as a rightmost child, the sequence is initialized to $C\left(R_{i}\right)=\boldsymbol{v}=(\operatorname{dep}(v), \operatorname{label}(v))$. 


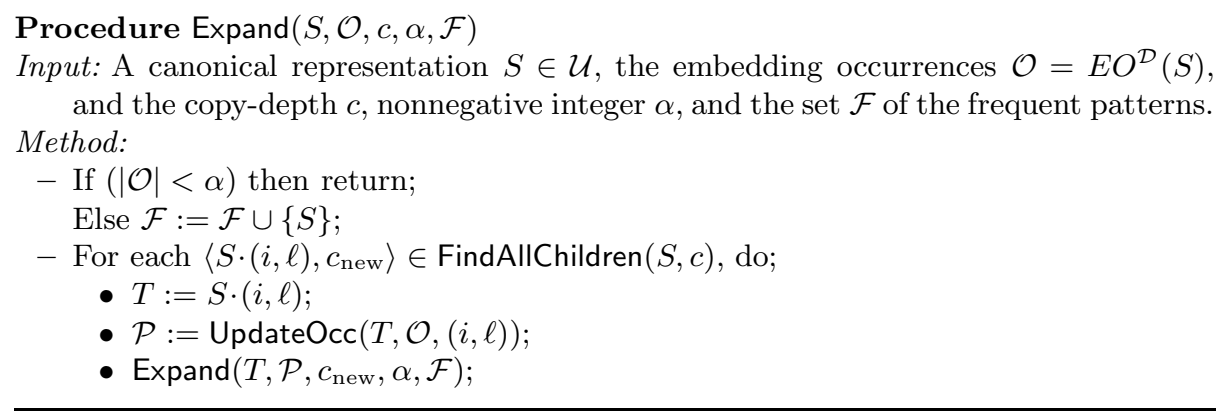

Fig. 6. A depth-first search procedure Expand

2. Whenever a new node $v$ of depth $d=\operatorname{dep}(v)>i$ comes to $T$, the right tree $R_{i}$ grows. In this case, $v$ is attached as the rightmost child of $r_{d-1}$. There are two cases below:

(i) Suppose that there exists a disagreement in $C\left(L_{i}\right)$ and $C\left(R_{i}\right)$. If $\boldsymbol{r}_{d e p(v)} \geq$ $\boldsymbol{v}$ then the rightmost expansion with $v$ does not violate the left-heavy condition of $T$, where $r_{d e p(v)}$ is the node preceding $v$ in the new tree.

(ii) Otherwise, we know that $C\left(R_{i}\right)$ is a prefix of $C\left(L_{i}\right)$. In this case, we say $R_{i}$ is copying $L_{i}$. Let $m=\left|C\left(R_{i}\right)\right|<\left|C\left(L_{i}\right)\right|$ and $\boldsymbol{w}$ be the $m$-th component of $C\left(L_{i}\right)$. For every new node $\boldsymbol{v}, T \cdot \boldsymbol{v}$ is a valid expansion if $\boldsymbol{w} \geq \boldsymbol{v}$ and $\boldsymbol{r}_{d e p(v)} \geq \boldsymbol{v}$. Otherwise, it is invalid.

(iii) In cases (i) and (ii) above, if $r_{d e p(v)-1}$ is a leaf of the rightmost branch of $T$ then $\boldsymbol{r}_{d e p(v)}$ is undefined. In this case, we define $\boldsymbol{r}_{d e p(v)}=T_{\infty}$.

3. Finally, $T$ reaches $C\left(L_{i}\right)=C\left(R_{i}\right)$. Then, the further rightmost expansion to $R_{i}$ is not possible.

If we expand a given unordered pattern $T$ so that all the right trees $R_{0}, \ldots, R_{g}$ satisfy the above conditions, then the resulting tree is in canonical form.

Let $R M B(T)=\left(r_{0}, r_{1}, \ldots, r_{g}\right)$ be the rightmost branch of $T$. For every $i=0,1, \ldots, g-1$, the internal node $r_{i}$ is said to be active at depth $i$ if $C\left(R_{i}\right)$ is a prefix of $C\left(L_{i}\right)$. The copy depth of $T$ is the depth of the highest active node in $T$. To deal with special cases, we introduce a trick: We define the leaf $r_{g}$ to be always active. Thus we have that if all nodes but $r_{g}$ are not active then its copy-depth is $g$. This trick greatly simplies the description of the update below.

Now, we explain how to generate all child trees of a given canonical representation $T \in \mathcal{C}$. In Fig. 7, we show the algorithm FindAllChildren that computes the set of all canonical child trees of a given canonical representation as well as their copy depths.

The algorithm is almost same as the algorithm for unlabeled unordered trees described in [12]. The update of the copy depth is slightly different from [12] by the existence of the labels. Let $T$ be a labeled ordered tree with the rightmost branch $R M B(T)=\left(r_{0}, r_{1}, \ldots, r_{g}\right)$ and the copy depth $k \in\{-1,0,1, \ldots, g-1\}$. Note that in the procedure FindAllChildren, the case where all but $r_{g}$ are inactive, including the case for chain trees, is implicitly treated in Case I.

To implement the algorithm FindAllChildren to enumerate the canonical child trees in $O(1)$ time per tree, we have to perform the following operation in $O(1)$ time: updating a tree, access to the sequence of left and right trees, maintenance 
Procedure FindAllChildren $(T, c)$ :

Method : Return the set $S u c c$ of all pairs $\langle S, c\rangle$, where $S$ is the canonical child tree of $T$ and $c$ is its copy depth generated by the following cases:

Case I : If $C\left(L_{k}\right)=C\left(R_{k}\right)$ for the copy depth $k$ :

- The canonical child trees of $T$ are $T \cdot\left(1, \ell_{1}\right), \ldots, T \cdot\left(k+1, \ell_{k+1}\right)$, where label $\left(r_{i}\right) \geq \ell_{i}$ for every $i=1, \ldots, k+1$. The trees $T \cdot\left(k+2, \ell_{k+2}\right), \ldots, T$. $\left(g+1, \ell_{g+1}\right)$ are not canonical.

- The copy depth of $T \cdot\left(i, \ell_{i}\right)$ is $i-1$ if $\operatorname{label}\left(r_{i}\right)=\ell_{i}$ and $i$ otherwise for every $i=1, \ldots, k+1$.

Case II : If $C\left(L_{k}\right) \neq C\left(R_{k}\right)$ for the copy depth $k$ :

- Let $m=\left|C\left(R_{k}\right)\right|+1$ and $\boldsymbol{w}=(d, \ell)$ be the $m$-th component of $C\left(L_{k}\right)$ (the next position to be copied). The canonical child trees of $T$ are $T \cdot\left(1, \ell_{1}\right), \ldots, T \cdot\left(d, \ell_{d}\right)$, where $\operatorname{label}\left(r_{i}\right) \geq \ell_{i}$ for every $i=1, \ldots, d-1$ and $\ell \geq \ell_{d}$ holds.

- The copy depth of $T \cdot\left(i, \ell_{i}\right)$ is $i-1$ if label $\left(r_{i}\right)=\ell_{i}$ and $i$ otherwise for every $i=1, \ldots, d-1$. The copy depth of $T \cdot\left(d, \ell_{d}\right)$ is $k$ if $\boldsymbol{w}=\boldsymbol{v}$ and $d$ otherwise.

Fig. 7. The procedure FindAllChildren

of the position of the shorter prefix at the copy depth, retrieval of the depth-label pair at the position, and the decision of the equality $C\left(L_{i}\right)=C\left(R_{i}\right)$.

To do this, we represent a pattern $T$ by the structure shown in Fig. 4.

- An array code : [1..size $] \rightarrow(\mathbf{N} \times \mathcal{L})$ of depth-label pairs that stores the depth-label sequence of $T$ with an integer size $\geq 0$ having the length of code.

- A stack $R M B:[0 . . t o p] \rightarrow(\mathbf{N} \times \mathbf{N} \times\{=, \neq\})$ of the triples (left, right, cmp). For each (left, right, cmp) $=R M B[i]$, left and right are the starting positions of the subsequences of code that represent the left tree $L_{i}$ and the right tree $R_{i}$, and the flag $\mathrm{cmp} \in\{=, \neq\}$ indicates whether $L_{i}=R_{i}$ holds. The length of the rightmost branch is top $\geq 0$.

It is not difficult to see that we can implement all the operation in FindAllChildren of Fig. 7 to work in $O(1)$ time, where an entire tree is not output but the difference from the previous tree. The proof of the next lemma is almost same to [12] except the handling of labels.

Lemma 6 (Nakano and Uno [12]). For every canonical representation $T$ and its copy depth $c \geq 0$, FindAllChildren of Fig. 7 computes the set of all the canonical child trees of $T$ in $O(1)$ time per tree, when only the differences to $T$ are output.

The time complexity $O(1)$ time per tree of the above algorithm is significantly faster than the complexity $O\left(k^{2}\right)$ time per tree of the straightforward algorithm based on Lemma 3, where $k$ is the size of the computed tree.

\subsection{Updating the Occurrence List}

In this subsection, we give a method for incrementally computing the embedding occurrences $E O^{\mathcal{D}}(T)$ of the child tree $T$ from the occurrences $E O^{\mathcal{D}}(S)$ of the canonical representation $S$.

In Fig. 8, we show the procedure UpdateOcc that, given a canonical child tree $T$ and the occurrences $E O^{\mathcal{D}}(S)$ of the parent tree $S$, computes its embedding occurrences $E O^{\mathcal{D}}(T)$. 


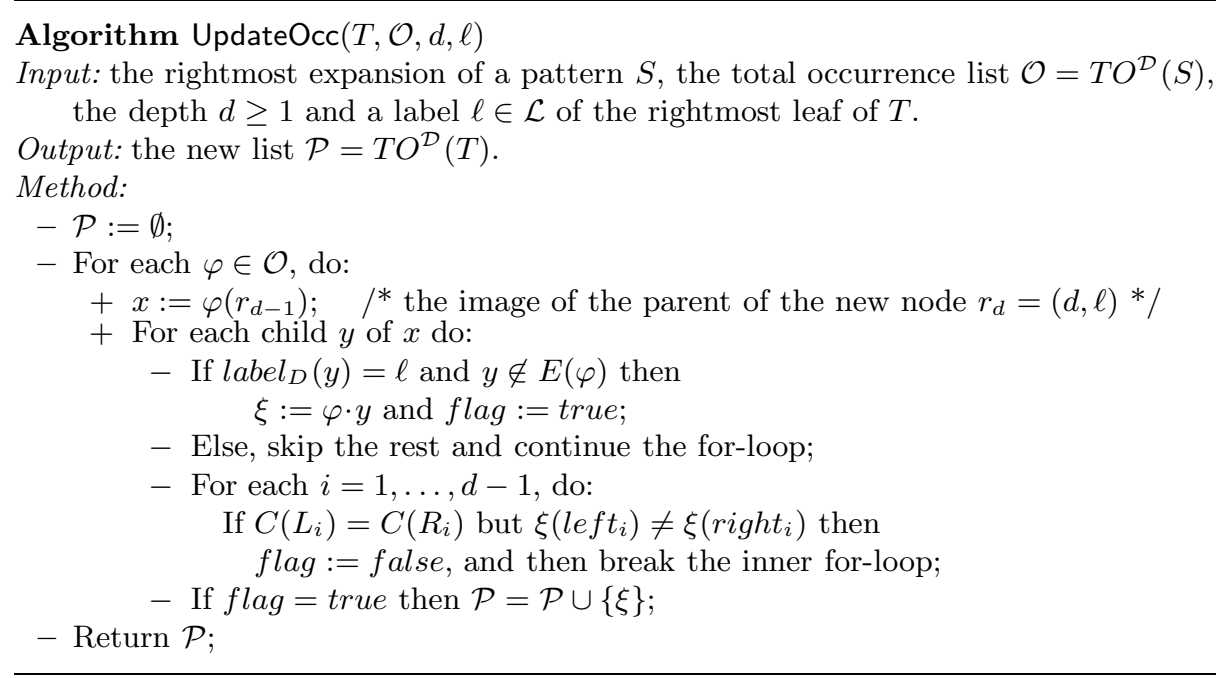

Fig. 8. An algorithm for updating embedding occurrence lists of a pattern

\subsection{Incremental Computation of Embedding Occurrences}

Let $T$ be a canonical representation for a labeled unordered tree over $\mathcal{L}$ with domain $\{1, \ldots, k\}$. Let $\varphi \in \mathcal{M}^{\mathcal{D}}(T)$ be a matching from $T$ into $\mathcal{D}$. Recall that the total and the embedding occurrences of $T$ associated with $\varphi$ is $\operatorname{TO}(\varphi)=$ $\langle\varphi(1), \ldots, \varphi(k)\rangle$ and $E O(\varphi)=\{\varphi(1), \ldots, \varphi(k)\}$, respectively. For convenience, we identify $\varphi$ to $T O(\varphi)$. Thus, we may write $\varphi(v)$ or $E O(\varphi)$ for a tuple $\varphi=$ $\left\langle v_{1}, \ldots, v_{k}\right\rangle \in V_{\mathcal{D}}^{k}$ in what follows.

We encode an embedding occurrence $E O$ in one of the total occurrences $\varphi$ corresponding to $E O=E O(\varphi)$. Since there are many total occurrences corresponding to $E O$, we introduce the canonical representation for embedding occurrences similarly as in Section 3.

Two total occurrences $\varphi_{1}$ and $\varphi_{2}$ are equivalent each other if $\operatorname{EO}\left(\varphi_{1}\right)=$ $\operatorname{EO}\left(\varphi_{2}\right)$. The occurrences $\varphi_{1}$ is heavier than $\varphi_{2}$, denote by $\varphi_{1} \geq_{\operatorname{lex}} \varphi_{2}$, if $\varphi_{1}$ is lexicographically larger than $\varphi_{2}$ as the sequences in $\mathbf{N}^{*}$. We give the canonical representation for the embedding occurrences.

Definition 4. Let $T$ be a canonical form of a labeled unordered tree and $E O \subseteq$ $V_{\mathcal{D}}$ be its embedding occurrence in $\mathcal{D}$ The canonical representation of $T$, denoted by $C R(E O)$, is the total occurrence $\varphi \in \mathcal{M}^{\mathcal{D}}(T)$ that is the heaviest tuple in the equivalence class $\left\{\varphi^{\prime} \in \mathcal{M}^{\mathcal{D}}(T) \mid \varphi^{\prime} \equiv \varphi\right\}$.

By Lemma 2, we can see that a straightforward generate-and-test algorithm requires $k^{O(k)}$ time to decide if a given total occurrence is the canonical representation for an embedding occurrence.

Let $\varphi=\langle\varphi(1), \ldots, \varphi(k)\rangle$ be an total occurrence of $T$ over $\mathcal{D}$. We denote by $P(\varphi)$ the unique total occurrence of length $k-1$ derived from $\varphi$ by removing the last component $\varphi(k)$. We say $P(\varphi)$ is a parent occurrence of $\varphi$ and $\varphi$ is a child occurrence of $P(\varphi)$.

Lemma 7. For any total occurrence $\varphi \in \mathcal{M}^{\mathcal{D}}(T)$, if $\varphi$ is in canonical form then so is its parent $P(\varphi)$. 


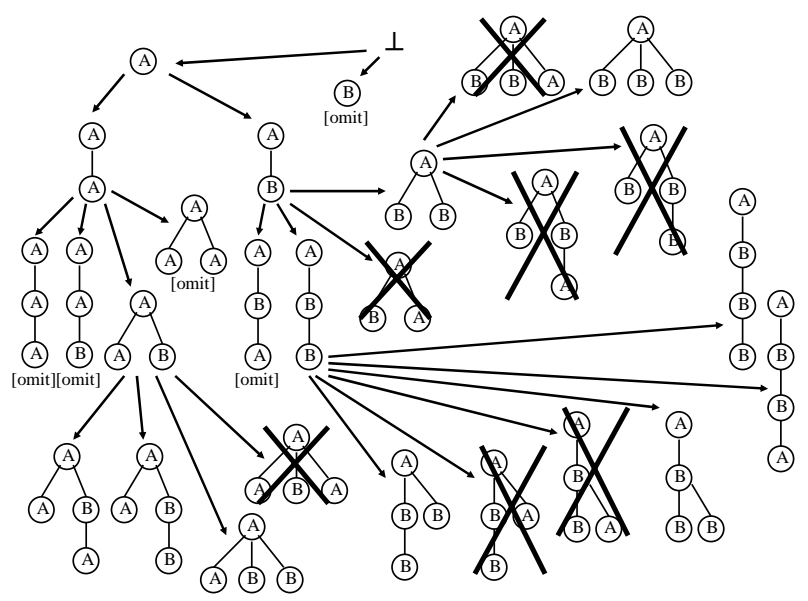

Fig. 9. A search tree for labeled unordered trees

The converse of Lemma 7 does not hold in general. Let $T$ be a labeled unordered tree, $\varphi \in \mathcal{M}^{\mathcal{D}}(T)$ be a total occurrence, and $v \in V_{T}$ be a node. Then, we denote by $\varphi(T(v))=\langle\varphi(i), \varphi(i+1), \ldots, \varphi(i+|T(v)|-1)\rangle$ the restriction of $\varphi$ to the subtree $T(v)$, where $\langle i, i+1, \ldots, i+|T(v)|-1\rangle$ is the nodes of $T(v)$ in preorder.

Lemma 8. Let $T$ be a labeled ordered tree and $\varphi \in \mathcal{M}^{\mathcal{D}}(T)$ be a total occurrence of $T$ in $\mathcal{D}$. Then, $\varphi$ is the canonical representation for an embedding occurrence iff $\varphi$ is partially left-heavy, that is, for any nodes $v_{1}, v_{2} \in V_{T}$, both of $\left(v_{1}, v_{2}\right) \in B$ and $T\left(v_{1}\right)=T\left(v_{2}\right)$ imply $\varphi\left(T\left(v_{1}\right)\right) \geq_{\operatorname{lex}} \varphi\left(T\left(v_{2}\right)\right)$.

First, we consider the incremental computation of the child total occurrences.

Lemma 9. Let $k \geq 1$ be any positive integer, $S$ be a canonical representation and $\phi$ be its canonical occurrence of $S$ in $\mathcal{D}$. For a node $w \in V_{\mathcal{D}}$, let $T=S \cdot \boldsymbol{v}$ be a child tree of $S$ with the rightmost branch $\left(r_{0}, \ldots, r_{g}\right)$. Then, the mapping $\phi=\varphi \cdot \boldsymbol{w}$ is a canonical total occurrence of $T$ in $D$ iff the following conditions (1)-(4) hold.

(1) label $_{D}(w)=$ label $_{T}(v)$.

(2) For every $i=1, \ldots, k-1, w \neq \varphi(i)$.

(3) $w$ is a child of $\varphi\left(r_{d-1}\right)$, where $r_{d-1} \in R M B(S)$ is the node of depth $d-1$ on the rightmost branch $R M B(S)$ of $S$.

(4) $C\left(L_{i}\right)=C\left(R_{i}\right)$ implies $\phi\left(\operatorname{root}\left(L_{i}\right)\right)=\phi\left(\operatorname{root}\left(R_{i}\right)\right)$ for every $i=0, \ldots, g-1$.

From Lemma 9, we present the procedure UpdateOcc of Fig. 8. The correctness of the procedure is obvious from Lemma 9 . To show the running time, we can see that the decision $C\left(L_{i}\right)=C\left(R_{i}\right)$ can be decidable in $O(1)$ time using a flag $c m p$ on the state and that the nodes $r_{d-1}$, left $t_{i}$ and right $t_{i}$ can be retrieved by the stack and the code array. Note that there is at least one canonical child tree among all the possibly non-canonical child trees obtained by the rightmost expansion of a canonical parent tree. Therefore, we have the following lemma.

Lemma 10. Let $S$ be a canonical representation of a unordered tree, $\mathcal{O}$ be its canonical total occurrences, and $T$ be a child tree of $S$ with the depth-label pair $(d, \ell) \in \mathbf{N} \times \mathcal{L}$. Then, the algorithm UpdateOcc of Fig. 8 computes the list of all the canonical total occurrences of $T$ in $O(k b m)$ time on the input $\langle T, \mathcal{O}, d, \ell\rangle$, where $k=|T|, b$ is the maximum branching factor in the database $\mathcal{D}$, and $m=$ $|\mathcal{O}|$ be the number of occurrences in $\mathcal{O}$. 
We show the main theorem of this paper.

Theorem 1. Let $\mathcal{D}$ be any unordered tree database and $\alpha \geq 0$ be any nonnegative integer. Then, the algorithm Unot of Fig. 5 enumerates all the canonical representations for the frequent unordered trees w.r.t. embedding occurrences without duplicates in $O\left(k b^{2} m\right)$ time per frequent patterns, where $b$ is the maximum branching factor in $\mathcal{D}, k$ is the maximum size of patterns enumerated, and $m$ is the number of embeddings of the enumerated pattern.

Proof. Combining Lemma 6 and Lemma 10, we see that the algorithm Unot computes frequent patterns in $O\left(\mathrm{kbm}^{\prime}\right)$ time per tree $T \in \mathcal{C}$, where $S=P(T)$ is a parent tree of $T$ and $m^{\prime}=|E O(S)|$ of the embedding occurrences of $S$. Since $|E O(T)|=O(b|E O(S)|)$, we have the result.

Fig. 9 illustrates the computation of the algorithm Unot that is enumerating a subset of labeled unordered trees of size at most 4 over $\mathcal{L}=\{A, B\}$. The arrows indicates the parent-child relation and the crossed trees are non-canonical ones.

\subsection{Comparison to a Straightforward Algorithm}

We compare our algorithm Unot to a straightforward algorithm Naive that uses a generate and test approach based on Lemma 3 of Section 3. Given a database $\mathcal{D}$ and a threshold $\sigma$, Naive runs as follows: Naive enumerates all the labeled ordered trees over $\mathcal{L}$ in $O(1)$ time using the rightmost expansion, and then for each tree it checks if it is in canonical form simply applying Lemma 3. Since there are $O(k|\mathcal{L}|)$ child patterns and the check takes $O\left(k^{2}\right)$ per tree, this stage takes $O\left(|\mathcal{L}| k^{3}\right)$ time per tree. It takes $O\left(n^{k}\right)$ time to compute all the embedding occurrences of $T$ in $\mathcal{D}$ by simply enumerating all $k$ combinations of the nodes in $\mathcal{D}$. Thus, the overall time is $O\left(|\mathcal{L}| k^{3}+n^{k}\right)$.

On the other hand, our algorithm computes the canonical representations in $O\left(k b^{2} m\right)$, where the total number $m$ of the embedding occurrences of $T$ is $m=O\left(n^{k}\right)$ in the worst case. However, $m$ will be much smaller than $n^{k}$ as the pattern size of $T$ grows. Thus, if it is the case that $b$ is a small constant and the occurrences size $m=|E O(T)|$ is much smaller than the total database size $n=\left|V_{\mathcal{D}}\right|$ then our algorithm will be faster than the straightforward algorithm.

\section{Conclusions}

In this paper, we presented an efficient algorithm Unot that computes all frequent labeled unordered trees appearing in a collection of data trees. This algorithm has a provable performance in terms of the output size unlike previous graph mining algorithms. It enumerates each frequent pattern $T$ in $O\left(k b^{2} n\right)$ per pattern, where $k$ is the size of $T, b$ is the branching factor of the data tree, and $n$ is the total number of occurrences of $T$ in the data trees.

We are implementing a prototype system of the algorithm and planning the computer experiments on synthesized and real-world data to give empirical evaluation of the algorithm in the revised version. The results will be included in the revised paper.

Some graph mining algorithms such as AGM [8], FSG [9], and gSpan [17] use various types of the canonical representation for general graphs similar to our canonical representation for unordered trees in Section 3. AGM [8] and FSG [9] employ the adjacent matrix with the lexicographically smallest row vectors under 
the permutation of rows and columns. gSpan [17] uses as the canonical form the DFS code generated with the depth-first search over a graph. It is a future problem to study the relationship among these techniques based on canonical coding and to develop efficient coding scheme for restricted subclasses of graph patterns.

\section{Acknowledgement}

Tatsuya Asai and Hiroki Arimura would like to thank Ken Satoh, Hideaki Takeda, Tsuyoshi Murata, and Ryutaro Ichise for the fruitful discussions on Semantic Web mining, and to thank Takashi Washio, Akihiro Inokuchi, Michihiro Kuramochi, and Ehud Gudes for the valuable discussions and comments on graph mining. Tatsuya Asai is grateful to Setsuo Arikawa for his encouragement and support for this work.

\section{References}

1. K. Abe, S. Kawasoe, T. Asai, H. Arimura, and S. Arikawa. Optimized Substructure Discovery for Semi-structured Data, In Proc. PKDD'02, 1-14, LNAI 2431, 2002.

2. Aho, A. V., Hopcroft, J. E., Ullman, J. D., Data Structures and Algorithms, Addison-Wesley, 1983.

3. T. R. Amoth, P. Cull, and P. Tadepalli, Exact learning of unordered tree patterns from queries, In Proc. COLT'99, ACM Press, 323-332, 1999.

4. T. Asai, K. Abe, S. Kawasoe, H. Arimura, H. Sakamoto, S. Arikawa, Efficient Substructure Discovery from Large Semi-structured Data, In Proc. the 2nd SIAM Int'l Conf. on Data Mining (SDM2002), 158-174, 2002.

5. T. Asai, H. Arimura, K. Abe, S. Kawasoe, S. Arikawa, Online Algorithms for Mining Semi-structured Data Stream, In Proc. the 2002 IEEE Int'l Conf. on Data Mining (ICDM'02), 27-34, 2002.

6. David Avis, Komei Fukuda, Reverse Search for Enumeration, Discrete Applied Mathematics, 65(1-3), 21-46, 1996.

7. L. B. Holder, D. J. Cook, S. Djoko, Substructure Discovery in the SUBDUE System, In Proc. KDD'94, 169-180, 1994.

8. A. Inokuchi, T. Washio, H. Motoda, An Apriori-Based Algorithm for Mining Frequent Substructures from Graph Data, In Proc. PKDD 2000, 13-23, LNAI, Springer, 2000.

9. M. Kuramochi, G. Karypis, Frequent Subgraph Discovery, In Proc. IEEE ICDM'01, 2001.

10. T. Miyahara, Y. Suzuki, T. Shoudai, T. Uchida, K. Takahashi, H. Ueda, Discovery of Frequent Tag Tree Patterns in Semistructured Web Documents, In Proc. PAKDD-2002, 341-355, 2002.

11. S. Nakano, Efficient generation of plane trees, Information Processing Letters, 84, 167-172, 2002.

12. S. Nakano, T. Uno, Another Simple Algorithm to Generate All Rooted Trees, 2003. (Submitting)

13. S. Nestrov, S. Abiteboul, R. Motwani, Extracting Schema from Semistructured Data, In Proc. SIGKDD'98, 295-306, 1998.

14. T. Uno, A Fast Algorithm for Enumerating Bipartite Perfect Matchings, In Proc. ISAAC 2001, LNCS, Springer-Verlag, 367-379, 2001.

15. N. Vanetik, E. Gudes, E. Shimony, Computing Frequent Graph Patterns from Semistructured Data, In Proc. IEEE ICDM'02, 458-465, 2002.

16. K. Wang, H. Liu, Schema Discovery from Semistructured Data, In Proc. KDD'97, 271-274, 1997.

17. X. Yan, J. Han, gSpan: Graph-Based Substructure Pattern Mining, In Proc. IEEE ICDM'02, 721-724, 2002.

18. M. J. Zaki. Efficiently Mining Frequent Trees in a Forest, In Proc. SIGKDD 2002, ACM, 2002. 